\title{
一种新颖的构建 $(E)-3$-芳氧基丙烯酸酯衍生物的方法
}

\author{
陈翠* 谭丽泉邱会华 \\ (广东石油化工学院化学工程学院 茂名 525000)
}

\begin{abstract}
摘要 以炔酸酯和酚类化合物为原料, $N, N$ 二甲基甲酰胺( $\mathrm{DMF}$ )为溶剂, $\mathrm{CuO} / \mathrm{CH}_{3} \mathrm{ONa}$ 为促进体系, 经过分子间的加成 和自由基反应, 成功地构建出了(E)-3-芳氧基丙烯酸酷衍生物. 采用 IR, ${ }^{1} \mathrm{H} \mathrm{NMR},{ }^{13} \mathrm{C} N \mathrm{NR}$ 和元素分析对目标产物进行 了表征. 研究表明该方法具有原料便宜易得、后处理操作简单和产品纯度高等优点.
\end{abstract}

关键词＼cjkstart炔酸酯; 酚类化合物; 分子间加成; $(E)$-3-芳氧基丙烯酸酯衍生物

\section{A Novel Construction Method for (E)-3-Aryloxyacrylate Derivatives}

\author{
Chen, Cui* Tan, Liquan Qiu, Huihua \\ (College of Chemcal Engineering, Guangdong University of Petrochemical Technology, Maoming 525000)
}

\begin{abstract}
This paper reports a novel intermolecular addition and free radical homolytic reaction for the synthesis of (E)-3-aryloxyacrylate derivatives from alkynoates and phenols using $N, N$-dimethylformamide (DMF) as the solvent and $\mathrm{CuO} / \mathrm{CH}_{3} \mathrm{ONa}$ as the system of promotion. The structures of product were confirmed by IR, ${ }^{1} \mathrm{H} N M R,{ }^{13} \mathrm{C}$ NMR and elemental analysis. The results showed that this synthetic route had the advantages of easy-obtained raw materials, simple work-up and high purity product.
\end{abstract}

Keywords alkynoates; phenols; intermolecular addition; (E)-3-aryloxyacrylates

丙烯酸酯是重要的高分子单体和基本有机化工原 料, 在精细化工的应用中占有相当重要的地位, 其系列 产品成千上万，几乎涉及到工业领域各个部门，广泛应 用于涂料、胶黏剂、塑料、纺织、造纸、橡胶、石油、 水处理、化纤、制革等行业. 以丙烯酸酯制得的高聚物 具有耐候、耐紫外光、耐水、耐热等特性，从而使其在 涂料、皮革、化纤、造纸等方面得以广泛应用. 同时，该 类化合物在学术上也是一类非常重要的有机原料, 被广 泛应用于有机合成中来构建新的 C- $\mathrm{C}, \mathrm{C}-$ Hetero 键以 及其它有用的有机中间体 ${ }^{[1 \sim 7]}$. 本文以炔酸酯和酚类化 合物为原料, 发展出了一种极其简便和新颖的构建 (E)-3-芳氧基丙烯酸酯衍生物方法(Eq. 1), 该类化合物 在有机合成是一类构建杂环化合物非常理想的前体化 合物 ${ }^{[8]}$. 关于该类化合物的合成, 梁永明课题组在 2006 年对其进行了报道, 不过它们是利用末端的炔酸酯与酚 类化合物在弱碱性条件下反应得到该类化合物 ${ }^{[9]}$.

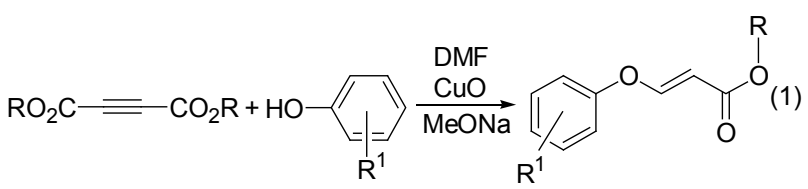

\section{1 结果与讨论}

\section{1 反应条件的优化}

首先，我们以丁炔二酸二乙酯(1a)和苯酚 (2a)反应 生成 $(E)$-3-苯氧基丙烯酸乙酯(3aa)为典型反应，考察了 反应时间、反应温度、溶剂、催化剂和碱的种类和用量 等方面对 3aa 产率的影响, 实验结果如表 1 所示. 从表 1 的数据可知, 在以甲苯、乙腈、1,2-二氯乙烷、1,4-二氧 六环作为溶剂的条件下, 该模型反应不发生(表 1 , Entries 1 4). 而当该反应在 $100{ }^{\circ} \mathrm{C}$ 的条件下进行时，以 $N, N$-二甲基甲酰胺(DMF)为溶剂时，反应 $4 \mathrm{~h}$ 能以 $25 \%$ 的收率得到目标化合物 3aa. 接下来，我们考察了不同 碱对该反应的影响, 研究发现甲醇钠和乙醇钠是比较理

\footnotetext{
*E-mail: cc161002@gmail.com

Received May 1, 2014; revised May 7, 2014; published online June 11, 2014.

Project supported by the Natural Science Foundation of Guangdong Province (No. S2012040007868) and the Excellent Young Scientist Fund of Guangdong Province Education Department (No.2013LYM_0059)..

广东省自然科学基金(No. S2012040007868)和广东省优秀青年基金(No. 2013LYM 0059)资助项目.
} 
想的碱, 它们相对于其它被篎选的碱而言, 更加有利于 此促进反应的进行(表 1 , Entries 5 10). 通过升高反应 温度以提高收率被证明是可行的(表 1, Entries 11, 12), 但是通过延长反应时间以提高收率被证明是不可取的 (表 1, Entries 12，13). 然后, 我们又探索了不同催化剂 对该反应的影响, 由实验结果可知, 氧化铜显示出了最 好的催化活性(表 1, Entries 14 19). 在此基础上, 我们 最后催化剂与碱的用量进行了进一步的考察, 发现氧化 铜的最佳用量为 $1.0,2.0$ equiv. 碱是最可取的(表 1, Entries $20 \sim 23)$. 该反应在此条件下进行, 通过 GC-MS 检 测，并未检测到酯交换产物.

\section{2 底物的拓展}

在最优化的反应条件下, 考察了丁炔二酸二乙酯和 丁炔二酸二甲酯与苯酚、邻甲基苯酚和间甲基苯酚的反 应, 结果见表 2 . 从表 2 中可知, 丁炔二酸二乙酯和丁炔 二酸二甲酯与苯酚、邻甲基苯酚和间甲基苯酚都能顺利 地反应，以中等到高的收率得到相应的产物. 带有甲基 取代的苯酚, 虽然也能使得该反应顺利进行, 但是, 相 对而言, 甲基取代在间位(2c)比甲基取代在邻位(2b)的
酚更适合于该反应体系, 并能以相对较高的收率得到相 应的目标化合物(表 2, Entries 2, 3, 5,6). 而丁炔二酸二 乙酯和丁炔二酸二甲酯针对于此反应是比较理想的反 应物, 这两类炔酸酯对该反应影响不大. 所有产物结构 经 IR, ${ }^{1} \mathrm{H}$ NMR, ${ }^{13} \mathrm{C} \mathrm{NMR}$ 和元素分析等进行了表征, 同 时, 我们根据烯碳氢的耦合常数确认了所有产物的结构 为反式结构. 为了拓宽反应的范围, 我们把该方法延伸 到苯丙炔酸乙酯、苯丙炔酸甲酯、丁炔酸甲酯、丁炔酸 乙酯和辛炔酸甲酯，但是，实验结果显示，所有这些单 炔酸酯都得不到预期的目标化合物.

\section{3 可能的催化反应机理}

到目前为止，我们对该反应的机理还没有完全弄清 楚. 在以往文献报道的基础上，以 $\mathbf{3 a a}$ 的形成为模型反 应对该反应提出了如 Scheme 1 所示的可能机理. 在碱 性条件下，炔酸酯首先与苯酚发生 Michael 加成反应， 然后加成产物与二价铜络合，得到中间体 $\mathbf{4}^{[8,10]}$. 在高温 的条件下，中间体 4 位阻大的一端的酯基以均裂的形式 断开, 得到自由基中间体 $\mathbf{5}$. 根据文献报道 ${ }^{[11 ~ 14], ~ D M F ~}$ 在强碱性和高温的条件下，很容易脱去醛基碳的氢，进

表 1 反应条件的优化 ${ }^{a}$

Table 1 Optimization of reaction conditions

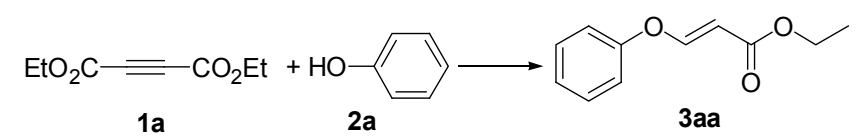

\begin{tabular}{|c|c|c|c|c|c|}
\hline Entry & Solvent & Reaction time/h & Temperature $/{ }^{\circ} \mathrm{C}$ & Additive/Alkali (equiv.) & Yield $^{b} / \%$ \\
\hline 1 & Toluene & 4 & 100 & $\mathrm{CuO} / \mathrm{MeONa}(1.0 / 2.0)$ & None \\
\hline 2 & $\mathrm{CH}_{3} \mathrm{CN}$ & 4 & Reflux & $\mathrm{CuO} / \mathrm{MeONa}(1.0 / 2.0)$ & None \\
\hline 3 & DCE & 4 & Reflux & $\mathrm{CuO} / \mathrm{MeONa}(1.0 / 2.0)$ & None \\
\hline 4 & Dioxane & 4 & 100 & $\mathrm{CuO} / \mathrm{MeONa}(1.0 / 2.0)$ & None \\
\hline 5 & DMF & 4 & 100 & $\mathrm{CuO} / \mathrm{MeONa}(1.0 / 2.0)$ & 25 \\
\hline 6 & DMF & 4 & 100 & $\mathrm{CuO} / \mathrm{AcONa}(1.0 / 2.0)$ & 11 \\
\hline 7 & DMF & 4 & 100 & $\mathrm{CuO} / \mathrm{K}_{2} \mathrm{CO}_{3}(1.0 / 2.0)$ & Trace \\
\hline 8 & DMF & 4 & 100 & $\mathrm{CuO} / \mathrm{KHCO}_{3}(1.0 / 2.0)$ & Trace \\
\hline 9 & DMF & 4 & 100 & $\mathrm{CuO} / \mathrm{KOH}(1.0 / 2.0)$ & 19 \\
\hline 10 & DMF & 4 & 100 & $\mathrm{CuO} / \mathrm{EtONa}(1.0 / 2.0)$ & 24 \\
\hline 11 & DMF & 4 & 120 & $\mathrm{CuO} / \mathrm{MeONa}(1.0 / 2.0)$ & 67 \\
\hline 12 & DMF & 4 & 140 & $\mathrm{CuO} / \mathrm{MeONa}(1.0 / 2.0)$ & 85 \\
\hline 13 & DMF & 6 & 140 & $\mathrm{CuO} / \mathrm{MeONa}(1.0 / 2.0)$ & 85 \\
\hline 14 & DMF & 4 & 140 & $\mathrm{CuCl}_{2} / \mathrm{MeONa}(1.0 / 2.0)$ & None \\
\hline 15 & DMF & 4 & 140 & $\mathrm{Cu}(\mathrm{OAc})_{2} / \mathrm{MeONa}(1.0 / 2.0)$ & None \\
\hline 16 & DMF & 4 & 140 & $\mathrm{Cu}_{2} \mathrm{O} / \mathrm{MeONa}(1.0 / 2.0)$ & 35 \\
\hline 17 & DMF & 4 & 140 & $\mathrm{CuBr} / \mathrm{MeONa}(1.0 / 2.0)$ & Trace \\
\hline 18 & DMF & 4 & 140 & $\mathrm{CuI} / \mathrm{MeONa}(1.0 / 2.0)$ & Trace \\
\hline 19 & DMF & 4 & 140 & $\mathrm{FeCl}_{3} / \mathrm{MeONa}(1.0 / 2.0)$ & Trace \\
\hline 20 & DMF & 4 & 140 & $\mathrm{CuO} / \mathrm{MeONa}(1.0 / 1.0)$ & 39 \\
\hline 21 & DMF & 4 & 140 & $\mathrm{CuO} / \mathrm{MeONa}(1.0 / 0.5)$ & 12 \\
\hline 22 & DMF & 4 & 140 & $\mathrm{CuO} / \mathrm{MeONa}(0.5 / 2.0)$ & 71 \\
\hline 23 & DMF & 4 & 140 & $\mathrm{CuO} / \mathrm{MeONa}(0.2 / 2.0)$ & 29 \\
\hline
\end{tabular}

${ }^{a}$ The reaction was carried out using $0.25 \mathrm{mmol}$ of 1a, $2.0 \mathrm{~mL}$ of solvent; ${ }^{b} \mathrm{GC}$ yield. 


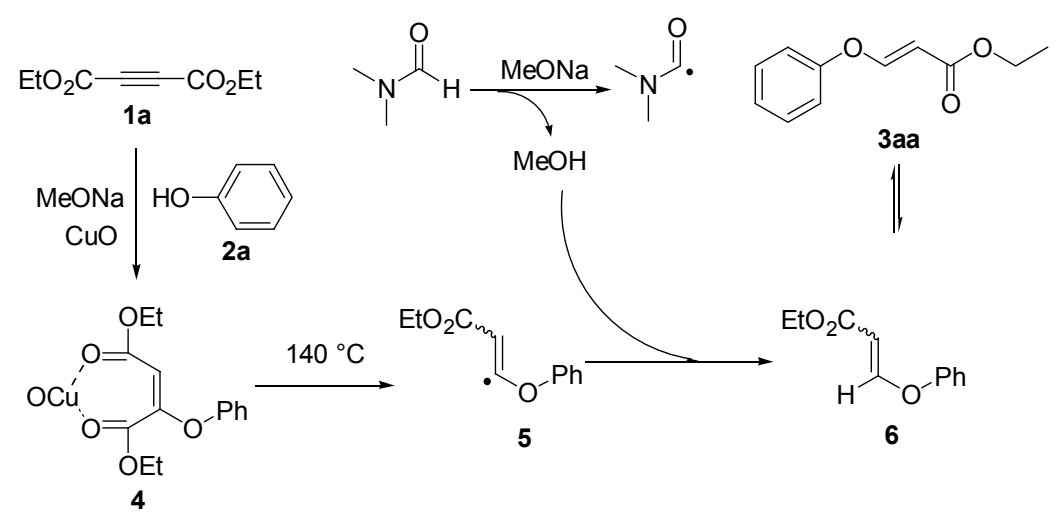

Scheme 1

表 2 底物的拓展 ${ }^{a}$

Table 2 Scope of the substrates

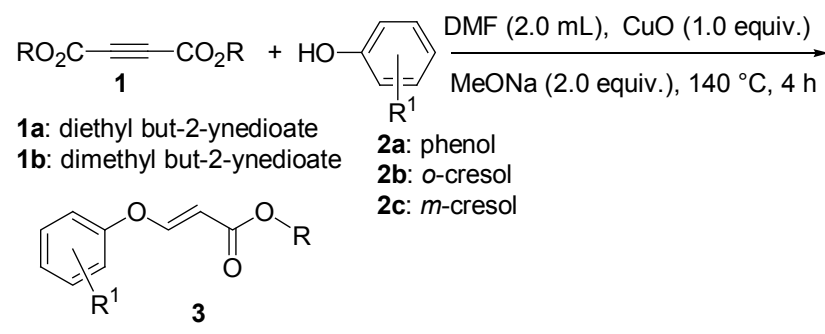

\begin{tabular}{ccccc}
\hline Entry & $\mathbf{1}$ & $\mathbf{2}$ & $\mathbf{3}$ & Yield $^{b} / \%$ \\
\hline 1 & $\mathbf{1 a}$ & $\mathbf{2 a}$ & $\mathbf{3 a a}$ & 80 \\
2 & $\mathbf{1 a}$ & $\mathbf{2 b}$ & $\mathbf{3 a b}$ & 69 \\
3 & $\mathbf{1 a}$ & $\mathbf{2 c}$ & $\mathbf{3 a c}$ & 76 \\
4 & $\mathbf{1 b}$ & $\mathbf{2 a}$ & $\mathbf{3 b a}$ & 81 \\
5 & $\mathbf{1 b}$ & $\mathbf{2 b}$ & $\mathbf{3 b b}$ & 72 \\
6 & $\mathbf{1 b}$ & $\mathbf{2 c}$ & $\mathbf{3 b c}$ & 77 \\
\hline
\end{tabular}

$\overline{{ }^{a}}$ Unless otherwise specified, all the reactions were carried out on $1.0 \mathrm{mmol}$ scale; ${ }^{b}$ Isolated yield.

而得到酰胺基自由基, 并伴随脱去一分子的醇. 自由基 5 夺取醇的质子进而生成中间体 $\mathbf{6}$, 该中间体经过异构 化转化为最稳定的异构体, 最终得到目标化合物 3aa.

\section{2 结论}

以 $\mathrm{CuO} / \mathrm{CH}_{3} \mathrm{ONa}$ 体系作为反应促进体系, 在 140 ${ }^{\circ} \mathrm{C}$ 和 $\mathrm{DMF}$ 作为溶剂的条件下, 通过炔酸酯与酚类化合 物 Michael 加成反应和高温条件下的自由基均裂反应, 高收率地实现了一系列 $(E)$-3-芳氧基丙烯酸酯衍生物的 合成. 此方法具有反应原料易得, 实验操作简单和产物 易分离等特点, 它为 $(E)$-3-芳氧基丙烯酸酯衍生物的合 成提供了一条新的途径.

\section{3 实验部分}

\section{1 仪器与试剂}

熔点采用 RD-II 型显微熔点仪测定; 红外光谱用 WGD-30/6 双光束红外分光光度计 $(\mathrm{KBr}$ 压片, 美国
Bruck 公司); 核磁共振谱用 Agilent400-MR 型核磁共振 仪(美国 Agilent 公司)测定, 氙代氯仿作溶剂, TMS 作内 标; 质谱用 Agilent1100 型气质联用仪(美国 Agilent 公 司)(GC/MSD VL ESI)测定; 紫外-可见吸收光谱用 WFH-204B 型手提式紫外分析仪测定; TLC 用板为 GF254(青岛海洋化工有限公司)高效薄层层析硅胶板. 实验所用试剂均购买于 Aldrich Chemicals 或 $\mathrm{J} \& \mathrm{~K}$ Scientific Ltd.

\section{$3.2(E)$-3-芳氧基丙烯酸酯衍生物合成的一般步骤}

在 $10 \mathrm{~mL}$ 的刻度试管中加入 DMF $2.0 \mathrm{~mL}, 1.0 \mathrm{mmol}$ 炔酸酯 $\mathbf{1}, 1.0 \mathrm{mmol}$ 酚 $2,1.0 \mathrm{mmol}$ 氧化铜、 $2.0 \mathrm{mmol}$ 甲 醇钠，在 $140{ }^{\circ} \mathrm{C}$ 的条件下搅拌反应 $4 \mathrm{~h}$. 反应结束后, 将反应体系冷却至室温, 将所得反应液经制备性薄层板 纯化得到产物 3 .

(E)-3-苯氧基-丙烯酸乙酯(3aa) ${ }^{[15]}$ : ${ }^{1} \mathrm{H} \mathrm{NMR}\left(\mathrm{CDCl}_{3}\right.$, $400 \mathrm{MHz}) \delta: 7.77(\mathrm{~d}, J=12.4 \mathrm{~Hz}, 1 \mathrm{H}), 7.35$ (t, $J=8.0 \mathrm{~Hz}$, 2H), $7.16(\mathrm{t}, J=7.2 \mathrm{~Hz}, 1 \mathrm{H}), 7.05(\mathrm{~d}, J=8.0 \mathrm{~Hz}, 2 \mathrm{H}), 5.53$ $(\mathrm{d}, J=12.4 \mathrm{~Hz}, 1 \mathrm{H}), 4.17(\mathrm{q}, J=7.2 \mathrm{~Hz}, 2 \mathrm{H}), 1.26$ (t, $J=$ $7.2 \mathrm{~Hz}, 3 \mathrm{H}) ;{ }^{13} \mathrm{C}$ NMR $\left(\mathrm{CDCl}_{3}, 100 \mathrm{MHz}\right) \delta: 167.3,159.0$, 156.1, 129.9, 124.9, 118.0, 102.1, 60.0, 14.3.

(E)-3-(邻甲基苯氧基)-丙烯酸乙酯(3ab): 浅黄色油 状物. ${ }^{1} \mathrm{H}$ NMR $\left(\mathrm{CDCl}_{3}, 400 \mathrm{MHz}\right) \delta: 7.75(\mathrm{~d}, J=12.4 \mathrm{~Hz}$, 1H), $7.18(\mathrm{t}, J=7.6 \mathrm{~Hz}, 2 \mathrm{H}), 7.06(\mathrm{t}, J=7.2 \mathrm{~Hz}, 1 \mathrm{H}), 6.96$ (d, $J=8.0 \mathrm{~Hz}, 1 \mathrm{H}), 5.34(\mathrm{~d}, J=12.4 \mathrm{~Hz}, 1 \mathrm{H}), 4.15$ (q, $J=$ $7.6 \mathrm{~Hz}, 2 \mathrm{H}$ ), 2.21 (s, 3H), 1.25 (t, $J=7.6 \mathrm{~Hz}, 3 \mathrm{H}) ;{ }^{13} \mathrm{C}$ NMR $\left(\mathrm{CDCl}_{3}, 100 \mathrm{MHz}\right) \delta: 167.3,161,153.7,131.5$, $129.0,127.3,125.3,118.5,101.0,60.0,15.8,14.3$; MS (EI) $\mathrm{m} / z$ (\%): 160.05 (100.00), 206.05 (70.55); IR (KBr) $v$ : 2958, 2924, 2859, 1711, 1635, 1589, 1572, 1450, 1383, $1243,1155,1098,798 \mathrm{~cm}^{-1}$. Anal. calcd for $\mathrm{C}_{12} \mathrm{H}_{14} \mathrm{O}_{3}: \mathrm{C}$ 69.88, H 6.84; found C 69.79, H 7.00.

(E)-3-(间甲基苯氧基)-丙烯酸乙酯(3ac)：浅黄色油 状物. ${ }^{1} \mathrm{H}$ NMR $\left(\mathrm{CDCl}_{3}, 400 \mathrm{MHz}\right) \delta: 7.79(\mathrm{~d}, J=12.0 \mathrm{~Hz}$, 
1H), $7.26(\mathrm{~d}, J=6.8 \mathrm{~Hz}, 1 \mathrm{H}), 7.01(\mathrm{~d}, J=7.6 \mathrm{~Hz}, 1 \mathrm{H}), 6.88$ (d, $J=8.0 \mathrm{~Hz}, 2 \mathrm{H}), 5.55$ (d, $J=12.0 \mathrm{~Hz}, 1 \mathrm{H}), 4.20$ (q, $J=$ $7.2 \mathrm{~Hz}, 2 \mathrm{H}), 2.37$ (s, 3H), $1.29(\mathrm{t}, J=7.2 \mathrm{~Hz}, 3 \mathrm{H}) ;{ }^{13} \mathrm{C}$ NMR $\left(\mathrm{CDCl}_{3}, 100 \mathrm{MHz}\right) \delta: 167.3,159.2,155.8,140.0$, 129.6, 125.7, 118.7, 115.0, 101.9, 60.0, 21.3, 14.3; IR (KBr) $v$ : 2956, 2853, 1716, 1650, 1600, 1585, 1456, 1372, 1250, 1149, 1113, 691, 711, $765 \mathrm{~cm}^{-1}$; MS (EI) $m / z$ (\%): 160.10 (100.00), 206.05 (40.30). Anal. calcd for $\mathrm{C}_{12} \mathrm{H}_{14} \mathrm{O}_{3}$ : C 69.88, H 6.84; found C 70.03, H 6.96.

(E)-3- 苯氧基一丙烯酸甲酯 (3ba) $)^{[10]}$ : ${ }^{1} \mathrm{H} \quad \mathrm{NMR}$ $\left(\mathrm{CDCl}_{3}, 400 \mathrm{MHz}\right) \delta: 7.79(\mathrm{~d}, J=12.4 \mathrm{~Hz}, 1 \mathrm{H}), 7.35(\mathrm{t}$, $J=7.6 \mathrm{~Hz}, 2 \mathrm{H}), 7.16(\mathrm{t}, J=7.2 \mathrm{~Hz}, 1 \mathrm{H}), 7.04$ (d, $J=8.0$ $\mathrm{Hz}, 2 \mathrm{H}), 5.53$ (d, $J=12.4 \mathrm{~Hz}, 1 \mathrm{H}), 3.71(\mathrm{~s}, 3 \mathrm{H}) ;{ }^{13} \mathrm{C} \mathrm{NMR}$ $\left(\mathrm{CDCl}_{3}, 100 \mathrm{MHz}\right) \delta: 167.8,159.2,155.8,129.9,125.0$, 118.0, 101.7, 51.3 .

(E)-3-(邻甲基苯氧基)-丙烯酸甲酯(3bb): 黄色油状 物. ${ }^{1} \mathrm{H}$ NMR $\left(\mathrm{CDCl}_{3}, 400 \mathrm{MHz}\right) \delta: 7.76(\mathrm{~d}, J=12.4 \mathrm{~Hz}$, 1H), 7.18 (t, $J=7.6 \mathrm{~Hz}, 2 \mathrm{H}), 7.08(\mathrm{t}, J=7.6 \mathrm{~Hz}, 1 \mathrm{H}), 6.96$ (d, $J=8.0 \mathrm{~Hz}, 1 \mathrm{H}), 5.37$ (d, $J=12.4 \mathrm{~Hz}, 1 \mathrm{H}), 3.69$ (s, 3H), 2.21 (s, $3 \mathrm{H}) ;{ }^{13} \mathrm{C}$ NMR $\left(\mathrm{CDCl}_{3}, 100 \mathrm{MHz}\right) \delta: 167.7,160.1$, 153.7, 131.5, 128.9, 127.3, 125.3, 118.4, 100.6, 51.2, 15.8; IR (KBr) v: 2963, 2871, 1715, 1611, 1607, 1580, 1465, 1377, 1250, 1115, 1098, $805 \mathrm{~cm}^{-1}$; MS (EI) $\mathrm{m} / z$ (\%): 160.10 (100.00), 192.05 (45.34). Anal. calcd for $\mathrm{C}_{11} \mathrm{H}_{12} \mathrm{O}_{3}$ : C 68.74, H 6.29; found C 68.77, H 6.47.

(E)-3-(间甲基苯氧基)-丙烯酸甲酯(3bc): 黄色油状 物. ${ }^{1} \mathrm{H}$ NMR $\left(\mathrm{CDCl}_{3}, 400 \mathrm{MHz}\right) \delta: 7.81(\mathrm{~d}, J=12.0 \mathrm{~Hz}$, 1H), $7.26(\mathrm{t}, J=7.2 \mathrm{~Hz}, 1 \mathrm{H}), 7.01$ (d, $J=7.6 \mathrm{~Hz}, 1 \mathrm{H}), 6.88$ (d, $J=8.0 \mathrm{~Hz}, 2 \mathrm{H}), 5.57$ (d, $J=12.0 \mathrm{~Hz}, 1 \mathrm{H}), 3.74(\mathrm{~s}, 3 \mathrm{H})$, 2.37 (s, 3H); ${ }^{13} \mathrm{C}$ NMR $\left(\mathrm{CDCl}_{3}, 100 \mathrm{MHz}\right) \delta: 167.7,159.3$,
155.8, 140.2, 129.6, 125.7, 118.6, 114.9, 101.5, 51.2, 21.3; MS (EI) $m / z$ (\%): 161.10 (100.00), 192.05 (95.63); IR (KBr) $v$ : 2933, 2869, 1661, 1495, 1439, 1390, 1255, 1101, $693,755,836 \mathrm{~cm}^{-1}$. Anal. calcd for $\mathrm{C}_{11} \mathrm{H}_{12} \mathrm{O}_{3}: \mathrm{C} 68.74, \mathrm{H}$ 6.29; found C 68.81, H 6.11 .

\section{References}

[1] McInturff, E. L. Mowat, J.; Waldeck, A. R.; Krische, M. J. J. Am. Chem. Soc. 2013, 135, 17230.

[2] Han, X. Y.; Wang, Y. Q.; Zhong, F. R.; Lu, Y. X. J. Am. Chem. Soc. 2011, 133, 1726.

[3] Anastasaki, A.; Nikolaou, V.; Zhang, Q.; Burns, J.; Samanta, S. R.; Waldron, C.; Haddleton, A. J.; McHale, R.; Fox, D.; Percec, V.; Wilson, P.; Haddleton, D. M. J. Am. Chem. Soc. 2014, 136, 1141.

[4] Percec, V.; Guliashvili, T.; Ladislaw, J. S.; Wistrand, A.; Stjerndahl, A.; Sienkowska, M. J.; Monteiro, M. J.; Sahoo, S. J. Am. Chem. Soc. 2006, 128, 14156.

[5] Walker, E. H.; Apblett, A. W.; Walker, R.; Zachary, A. Chem. Mater. 2004, 16, 5336.

[6] Xu, Y. H.; Lu, J.; Loh, T. P. J. Am. Chem. Soc. 2009, 131, 1372.

[7] Shirai, M.; Mitsukura, K.; Okamura, H. Chem. Mater. 2008, 20, 1971.

[8] Tan, P.; Li, N. B.; Xu, X. H. Chin. J. Org. Chem. 2012, 32, 2162.

[9] Fan, M. J.; Li, G. Q.; Li, L. H.; Yang, S. D.; Liang, Y. M. Synthesis 2006, 2286.

[10] Han, Y.; Sun, J.; Sun, Y.; Gao, H.; Yan, C. G. Chin. J. Org. Chem. 2012, 32, 1577.

[11] Øpstad, C. L.; MelØ, T. B.; Sliwka, H. R.; Partali, V. Tertrahedron 2009, 65, 7616.

[12] Chen, Y. Y.; Zhang, X. J.; Yuan, H. M.; Wei, W. T.; Yan, M. Chem. Commun. 2013, 49, 10974.

[13] Wei, W. T.; Dong, X. J.; Nie, S. Z.; Chen, Y. Y.; Zhang, X. Z.; Yan, M. Org. Lett. 2013, 15, 6018.

[14] Li, C. L.; Zhang, Y. C.; Li, P. H.; Wang, L. J. Org. Chem. 2011, 76, 4692.

[15] Kinart, W. J.; Kinart, A. Appl. Organomet. Chem. 2007, 21, 373. 